\title{
Consciousness and life after death in the evolution of intelligence
}

\author{
K.L. Senarath Dayathilake \\ Human Well-being Science Program, 177/1 A, Epitamulla Road, Kotte-10100, Sri Lanka \\ Correspondence Email: klsenarathdayathilake@yahoo.com
}




\title{
Consciousness and life after death in the evolution of intelligence
}

\begin{abstract}
:
To date, no scientific study has found evidence of an afterlife, and the mechanism of consciousness is two of the most challenging questions. Here, I show a hypothesis for consciousness and the probability of an afterlife through three simple thought experiments and theoretical evidence. More studies are needed to understand the mechanism precisely. I found that consciousness can be discussed based on a new theory. Here, I hypothesize that when a person or animal dies, the selection of a new nervous system characteristic of a new life might depend on the characteristics of the final evolved yet unknown particle. Here, I suggest that the particle's positive or adverse evolution depends on the natural evolution of the materialistic brain's cognition, including intelligence. The most intelligent persons with a higher potential scan mind virus may survive happier and help others improve their psychological well-being in the evolution of intelligence. When a brain dies, the two microparticles might emit at infinite speed from the dead brain and simultaneously bond with a naturally select suitable zygote or early nervous system somewhere in the universe/s, forming a new life with the impact of new nurture.
\end{abstract}




\section{Keywords}

Cognitive psychology, determinism, materialism, meditation, mind viruses, new physics, philosophy, theoretical hypothesis, thought experiment, ultraquantum particles.

Consciousness implies awareness: subjective, phenomenal experience of internal and external worlds; however, what consciousness remains unknown and plays an intrinsic role in the universe. (Hameroff \& Penrose 2014). In summary, science/materialism with consciousness has no distinctive role (Chalmers, 2012; Dennett, 1991; Dennett, 1995; Dennett \& Kinsbourne, 1992; Wegner, 2002), for example, dualism'spirituality, with consciousness outside of science (Berkeley, 1975; Chopra, 2001; Kant, 1998). Science with consciousness as an essential ingredient of physical law still needs to be fully understood. (Hameroff, 1998; Hameroff, 1998.; Hameroff, 2007; Hameroff \& Penrose, 1996; Hameroff \& Penrose, 1996; Penrose \& Hameroff, 1995; Penrose \& Hameroff, 2011; Whitehead, 1929; Whitehead, 1933; Zeman, 2008). How can we define consciousness? Is there a probability of an afterlife? How does matter and the new physics of the brain base on the origin of consciousness? These are out of three essential and unresolved questions on the life of 


\section{CONSCIOUSNESS AND AFTERLIFE}

the brain. Some say that consciousness is not a scientific term and lacks a technical definition, and we are learning to make sense of ourselves without invoking supernatural power ${ }^{19}$. Most scientists put aside the afterlife question, considering it a just religious and metaphysical belief. Near-death experience represents a biological paradox that challenges our understanding of the brain and has been advocated as evidence for life after death and the noncorporeal basis of human consciousness. (Alexander, 2012; Chopra, 2006; Long \& Perry, 2010; Thonnard, et al., 2013; van Lommel, 2010) It is based on an unsupported belief that the brain cannot be the source of highly vivid and lucid conscious experiences during clinical death. (Facco and Agrillo, 2012; Thonnard, et al. 2013; Mobbs \& Watt, 2011; van Lommel, 2011)

Nevertheless, the evidence thus far suggests that in the first few minutes after death, consciousness is not annihilated ${ }^{28}$. While many such studies' approaches are on near-death experiences, my methodology differs from those studies and is a new theoretical approach. This study on the theme was encouraged by researchers who revived disembodied pig brains and challenged definitions of life and death (Vrselja et al., 2019).

To philosophers, introspection and phenomenality seem independent or dissociable, although this is controversial. (Sutherland, 1989). The term 'consciousness' has four main topics: knowledge in general, intentionality, introspection (and the knowledge it generates), and phenomenal experience. 


\section{CONSCIOUSNESS AND AFTERLIFE}

On the other hand, some biophysicists handle the issue of consciousness in a multidisciplinary way. However, when a scientific inquiry into the brain and consciousness occurs, considerable knowledge of physical theories of the matters in the universe and its psychology is unavoidable. Neither general relativity nor quantum mechanics help discover these significant problems. When questioning whether there is a unified theory for everything, I found three possibilities: (a) there is a completely unified theory, (b) there is no such ultimate theory or just infinite sequence, and (c) no theory of universe and event cannot be predicted beyond a certain extent (Hawking, 2006). In other words, we cannot conclude universal theory precisely. Moreover, considering the knowledge of the brain and physical functions, free will is an illusion that shares common cognitive elements with paranormal beliefs' (Mogi, 2014)

Hawking told the Guardian, "There is no heaven or afterlife for broken down computers; that is a fairy story for people afraid of the dark." He believed the brain is like a computer that will shut off and regards the brain as a computer that will stop working when its components fail. (Hawking, 2011. Moreover, the stream of consciousness thoughts is naturally programmed by mind virus vs. healthy mind virus (MV vs. HMV) selection and neutral mind viruses (Dayathilake, 2017; Dayathilake, 2017; Dayathilake, 2017: Dayathilake, 2018). Here, the nature and nurturing of the brain may result in consciousness. Consciousness may result from multifactorial complex natural neuronal reflexes such as a network of the brain's nature, nurture, X-ultraquantum consciousness unique particle (X-UQCUP), and X-ultraquantum consciousness genomic particle (X-UQCGP); therefore, there is no free will. According to Theravada, Bainham outlines twenty-four kinds of 
conditional relation relations (Karunadasa, 2010) in the processes subject to relation (Gombrich, 2009) and no self that no unchanging, permanent self or essence can be found in any phenomenon (Machin, 2013).

Therefore, we still do not have a fundamental theory to explain the objectives of the article thus far, and I assume that an interdisciplinary study with a theoretical model may be helpful to initially find possible evidence of the issues of consciousness and the afterlife.

\section{Methods and materials}

The three theoretical experiments assumed that all participants had healthy brains and minds in similar environments. I assumed the first and third experiments were valid if cell death attenuated and preserved anatomical and neural cell integrity (Vrselja, Z. et al. 2019). From $T_{1}$ to $T_{2}$, six brains were dead; therefore, there was no consciousness. 


\section{CONSCIOUSNESS AND AFTERLIFE}

The participants in the three experiments were categorized into three groups:

I. The identical triplet participants include I-myself-me as 'a', you' as 'b,' and you $^{2}$ as 'c.' In other words, any article reader may assume he is a participant with two identical sublimes, as in identical triplets.

II. The second identical (triplet) participants were labeled he ${ }^{1}$ as 'd', he ${ }^{2}$ as 'e,' and $\mathrm{he}^{3}$ as person 'f,'

III. The nonidentical triplet is labeled 'g,' 'h,' and 'i.'

\section{Experiment 1}

All matters and functions from atoms, molecules, and neurons to the whole brain were identical in each triplet of I and II. Nutrients were given a similar quantity and quality, so their physiological, psychological, and physical processes could be identical and simultaneous; in other words, groups I, II, and III were nurtured similarly. I assumed that all similar (but not unique) subatomic particles, atoms of elements, in all brains were qualitatively and quantitatively identical and similarly functional according to quantum theory; similar chemical compounds in the brain behave similarly to theories in chemistry.

At age 18 , at $\mathrm{T}_{1}$, healthy persons of $\mathrm{a}, \mathrm{b}, \mathrm{d}, \mathrm{e}, \mathrm{g}$, and $\mathrm{h}$ were simultaneously killed without harming their brains. Postmortem samples of disembodied brains were kept in the laboratory until $\mathrm{T}_{2}$ using 


\section{CONSCIOUSNESS AND AFTERLIFE}

preservation technology (Vrselja, Z. et al. 2019). Over time, $\mathrm{T}_{2}$ simultaneously gives life to all dead brains.

\section{Results}

Whether identical or nonidentical, no one experiences their consciousness as nonunique, overlaps, coincides, or feels aware that a specific person is simultaneously in two or many environments at any given moment. Therefore, before $\mathrm{T}_{1}$, all nine participants' growth was independent, and consciousness streams might be distinct for each participant.

Soon after $\mathrm{T}_{1}$, the brains of $\mathrm{a}, \mathrm{b}, \mathrm{d}, \mathrm{e}, \mathrm{g}$, and $\mathrm{h}$ had no consciousness and were dead brains in the lab. However, c, f, and i live in the lab from birth to beyond time $\mathrm{T}_{2}$.

\section{Discussion}

What happened to the consciousness of $a, b, d, e, g$, and $h$ after $T_{1}$ ? For example, those whose consciousness lived as 'a' ( $\mathrm{T}_{1}$ to $\mathrm{T}_{3}$ ) and ' $\mathrm{b}$ ' $\mathrm{T}_{1}$ to $\mathrm{T}_{4}$ in the laboratory before $\mathrm{T}_{1}$ ? However, scientists are probably in trouble confirming whether similar consciousness of a, b, d, e, g, and h who lived until $\mathrm{T}_{1}$ (before the frozen) will live after $\mathrm{T}_{1}$ until $\mathrm{T}_{2}$ (see Venn diagram). I assumed their cognitive evolution might be shown in the second Venn diagram. (Here, I demonstrate that $\mathrm{a}, \mathrm{b}$, and $\mathrm{c}$ are just three examples of nine live brains.)

As shown in Venn diagram one, cognition (only considered for $a, b$, and c) 
$\mathrm{a} \cap \mathrm{b} \cap \mathrm{c}=\mathrm{X}_{1}$ or $\mathrm{a}, \mathrm{b}$ and $\mathrm{c}$ have similar cognition from $\mathrm{T}_{0}$ and $\mathrm{T}_{1}$

$d \cap e \cap f=X_{2}$ or in other words, $d, e$, and $f$ have similar cognition from $T_{0}$ and $T_{1}$

Cognition of $\mathrm{g}, \mathrm{h}$, and i will be

$\mathrm{g} \cap \mathrm{h} \cap \mathrm{i}=\varnothing$

\section{Experiment 2}

Suppose the whole-brain matter of a, b, d, e, g, and h were instantly separated to the atomic level at $\mathrm{T}_{1}$; moreover, after the six brains were simultaneously reconstructed at $\mathrm{T}_{2}$, these brains lived similar to those until $\mathrm{T}_{1}$ and similarly nurtured. The second experiment was designed to avoid error if the six brains in experiment one were not dead but had little consciousness, in other words, if they were in a nearly dead stage (yet not dead brains), and to minimize the error of quantum entanglement between the six individual brains when they gained consciousness at $T_{2}$.

\section{Result}




\section{CONSCIOUSNESS AND AFTERLIFE}

Suppose this experiment is theoretically acceptable; simultaneously, reconstructed brains of a, b, $\mathrm{d}$, e, g, and $\mathrm{h}$ will function from $\mathrm{T}_{2}$ and beyond as in experiment one. Furthermore, all identical brain volumes, anatomy, and physiological activities were similar in the laboratory, as in experiment one.

\section{Discussion}

A similar discussion may apply here, as in experiment two. (See Venn diagrams one and two)

\section{Experiment 3}

I suppose all identical twins and nonidentical triplicates were nurtured similarly to experiment one. The dead brains of $\mathrm{a}, \mathrm{b}, \mathrm{d}, \mathrm{e}, \mathrm{g}$, and $\mathrm{h}$ were frozen from $\mathrm{T}_{1}$ to $\mathrm{T}_{2}$ using preservation technology (Vrselja, Z. et al. 2019). I assumed they used a similar methodology to create twenty-seven new brains from elements, as mentioned in experiment two. These twenty-seven brains constructed materialistically similar triplicates of a, b, c, d, e, f, g, h, and i. Therefore, twenty-seven new participant brains at $T_{2}$ were $a^{1}, a^{2}, a^{3}, b^{1}, b^{2}, b^{3}, c^{1}, c^{2}, c^{3}, d^{1}, d^{2}, d^{3}, e^{1}, e^{2}, e^{3}, f^{1}, f^{2}, f^{3}, g^{1}, g^{2}, g^{3}$, $h^{1}, h^{2}, h^{3}, i^{1}, i^{2}$, and $i^{3}$. In addition to regaining the life of six frozen brains of $a, b, d, e, g$, and $h$ at $\mathrm{T}_{2}$. Therefore, thirty-six participants in the third experiment (including $\mathrm{c}, \mathrm{f}$, and $\mathrm{i}$, who continued their life from $\mathrm{T}_{0}$ and beyond $\mathrm{T}_{2}$ ) were in the lab from $\mathrm{T}_{2}$ onward. Hence, the living brains were at 


\section{CONSCIOUSNESS AND AFTERLIFE}

time $\mathrm{T}_{2}$ 'a' to $\mathrm{c}^{3}\left(\mathrm{a}, \mathrm{a}^{1}, \mathrm{a}^{2}, \mathrm{a}^{3}, \mathrm{~b}, \mathrm{~b}^{1}, \mathrm{~b}^{2}, \mathrm{~b}^{3}, \mathrm{c}, \mathrm{c}^{1}, \mathrm{c}^{2}\right.$, and $\left.\mathrm{c}^{3}\right), \mathrm{d}^{\prime}$ to $\mathrm{f}^{3}\left(\mathrm{~d}, \mathrm{~d}^{1}, \mathrm{~d}^{2}, \mathrm{~d}^{3}, \mathrm{e}, \mathrm{e}^{1}, \mathrm{e}^{2}, \mathrm{e}^{3}, \mathrm{f}, \mathrm{f}^{1}, \mathrm{f}^{2}\right.$, and $\left.\mathrm{f}^{3}\right), \mathrm{g}^{\prime}$ to $\mathrm{g}^{3}\left(\mathrm{~g}, \mathrm{~g}^{1}, \mathrm{~g}^{2}\right.$, and $\left.\mathrm{g}^{3}\right), \mathrm{h}$ to $\mathrm{h}^{3},\left(\mathrm{~h}, \mathrm{~h}^{1}, \mathrm{~h}^{2}\right.$, and $\left.\mathrm{h}^{3}\right)$, and $\mathrm{i}$ to $\mathrm{i}^{3}\left(\mathrm{i}, \mathrm{i}^{1}, \mathrm{i}^{2}\right.$, and $\left.\mathrm{i}^{3}\right)$. Therefore, brains

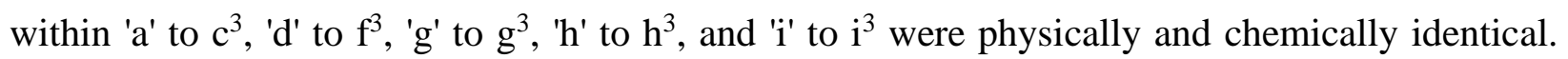
Human cloning is the closest empirical approach to these thought experiments, although they are not ethical and not perfectly applicable due to the lack of present science and biotechnology.

\section{Results}

If the third thought experiment was theoretically acceptable, I proposed that all twenty-two artificially built brains and the six frozen brains might live. Therefore, all thirty-three brain functions will simultaneously start at $\mathrm{T}_{2}$ and beyond, along with already continuously functioning three live brains of $\mathrm{c}, \mathrm{f}$, and $\mathrm{i}$ in the lab.

\section{Discussion}

However, no researcher would externally observe that (for example, here I considered a, b, and c out of six dead participants) 'I am/myself/me' - (participant 'a'), or/and you ${ }^{1}$ (b), and you ${ }^{2}$ (c) are out of the lab after $T_{1}$, who were indeed at the lab before $T_{1}$. Assuming three of the original participants' consciousness in the lab at $\mathrm{T}_{2}$ after gaining consciousness out of eleven identical brains of ' $a^{\prime}, a^{1}, a^{2}, a^{3}, b, b^{1}, b^{2}, b^{3}, c^{1}, c^{2}$, and $c^{3}$, it is not logical. What happened to their conscious minds before $T_{1}$ ? (See Venn diagrams one and two). Does their consciousness destroy forever? 
How can we say that their mind is destroyed without an afterlife? Significant questions remain.

Table 1. Results of experiments 1 to 3: cognitive function and consciousness of participants

Experiments:

$\mathbf{T}_{\mathbf{0}}$ to $\mathbf{T}_{1}$

$\mathbf{T}_{1}$ to $\mathbf{T}_{2}$

After $T_{2}$

\section{$\underline{\text { Experiment 1 }}$}

Cognitive functions of $\mathbf{a}, \mathbf{b}$, Similar and $\mathrm{c}$

Cognitive functions of

d, e, and $\mathbf{f}$
Life of $\mathbf{c}$ evolving in the $\mathbf{a}$ and $\mathbf{b}$ have similar $1 \mathrm{ab}$ cognition; $\mathbf{c}$ is older than a and b brains; Therefore, c's cognition is different from $\mathbf{a}$ and $\mathbf{b}$

Life $\mathbf{f}$ evolving in the lab $\mathbf{d}$ and $\mathbf{e}$ brains have similar cognition; $\mathbf{f}$ is 
older than $\mathbf{d}$ and $\mathbf{e}$; therefore, the cognition of ' $\mathbf{f}$ ' is different from $\mathbf{d}$ and $\mathbf{e}$

Cognitive functions of $\mathbf{g}, \mathbf{h}$, Different cognitions and $\mathbf{i}$

The Consciousness

Of all nine brains('a' to 'i')
All the original nine Streams

of Unique streams of frozen consciousnesses streams consciousness of $\mathbf{c}, \mathbf{f}$, brains of $\mathbf{a}, \mathbf{b}, \mathbf{d}, \mathbf{e}, \mathbf{g}$, were in the lab, unique ant and independent.

and ' $i$ ' were unique and and $h$ whose independent (the big consciousness before $T_{1}$
Life of $\mathbf{i}$ evolving in the lab cognition; 'i' is older than the other brains. question is what might not be in the lab. happened to those (What happened to $\mathbf{a}, \mathbf{b}$, original consciousness $\mathbf{d}, \quad \mathbf{e}, \mathbf{g}, \quad$ and $\mathbf{h}$ streams of $\mathbf{a}, \mathbf{b}, \mathbf{d}, \mathbf{e}, \mathbf{g}$, consciousnesses who and $h$ who were until originally lived until T) $\mathrm{T}_{1}$ ?)

Experiment 2 A similar result as in the Similar results as in Similar results and 
experiment one

\section{$\underline{\text { Experiment } 3}$}

Cognition of: $\mathbf{a}, \mathbf{a}^{\mathbf{1}}, \mathbf{a}^{\mathbf{2}}, \mathbf{a}^{\mathbf{3}}, \mathbf{b}, \mathbf{a}, \mathbf{b}$, and $\mathbf{c}$ similar $\mathbf{b}^{1}, \mathbf{b}^{2}, \mathbf{b}^{3}, \mathbf{c}, \mathbf{c}^{1}, \mathbf{c}^{2}$ cognitions

\section{And c3}

c still lives

c is still alive; frozen

(Then, what happened to the original brains of $\mathbf{a}$ ? and $\mathbf{b}$ ? Gain life in the lab. The rest of the newest brains of 
consciousness of frozen $\mathbf{a}^{1}, \mathbf{a}^{2}, \mathbf{a}^{3}, \mathbf{b}^{1}, \mathbf{b}^{2}, \mathbf{b}^{3}, \mathbf{c}^{1}, \mathbf{c}^{2}$, $\mathbf{a}$ and $\mathrm{b}$, who were until and $\mathbf{c}^{\mathbf{3}}$, and $\mathbf{a}$ ? and $\mathbf{b}$ ? $\mathbf{T}_{1}$ ?) have similar cognition. (What happened to the cognition of $\mathbf{a}$ and $\mathbf{b}$ in the lab before $\mathbf{T}_{\mathbf{1}}$ ?)
Cognitive function of similar $\mathbf{d}$, e, and $\mathbf{f}$ have similar brains of

$d, d^{1}, d^{2}, d^{3}, e, e^{1}, e^{2}, e^{3}$

$\mathbf{f}, \mathbf{f}^{\mathbf{1}}, \mathbf{f}^{\mathbf{2}}$, and $\mathbf{f}^{\mathbf{3}}$

cognitions
' $\mathbf{f}$ ' still alive in the lab (What happened to the original consciousness of frozen $\mathbf{d}$ and e those who lived until $\mathbf{T}_{\mathbf{1}}$ ?) f still alive in the lab; frozen brains of $\mathbf{d}$ ? and e? gained life; all nine newest brains of $\mathbf{d}^{\mathbf{1}}, \mathbf{d}^{\mathbf{2}}$, $\mathbf{d}^{\mathbf{3}}, \mathbf{e}^{\mathbf{1}}, \mathbf{e}^{\mathbf{2}}, \mathbf{e}^{\mathbf{3}}, \mathbf{f}^{\mathbf{1}}, \mathbf{f}^{\mathbf{2}}, \mathbf{f}^{\mathbf{3}}$ as well as d and e have similar cognition. (what happened to the consciousnesses of $\mathbf{d}$ 
and e, who were originally in the lab before $\mathbf{T}_{\mathbf{1}}$ ?)

g to $\mathbf{g}^{\mathbf{3}}$ have similar Cognitive function of $\mathbf{g}, \mathbf{g}^{\mathbf{1}}$, The cognitive functions 'i' still live (what cognition; $\mathbf{h}$ to $\mathbf{h}^{\mathbf{3}}$ have $\mathbf{g}^{\mathbf{2}}, \mathbf{g}^{\mathbf{3}}, \mathbf{h}, \mathbf{h}^{\mathbf{1}}, \mathbf{h}^{\mathbf{2}}, \mathbf{h}^{\mathbf{3}}, \mathbf{i}, \mathbf{i}^{\mathbf{1}}, \mathbf{i}^{\mathbf{2}}$, and of $\mathbf{g}, \mathbf{h}$, and $\mathbf{i}$ were happened to the original similar cognition, and $\mathbf{i}^{\mathbf{1}}$ $\mathbf{i}^{3}$ different consciousness of froze to $\mathbf{i}^{\mathbf{3}}$ have similar g and $\mathbf{h}$, those who lived cognition. The brain ' $\mathrm{i}$ ' is until T1?) older than the other eleven brains and has different cognition. What happened to the original consciousness of $\mathbf{g}$ and $\mathbf{h}$ ?

The consciousness of thirty- The nine original brains Unique consciousness All thirty-six live brains six brains of a to $\mathbf{i}^{\mathbf{3}} \quad$ in the lab had unique and streams of $\mathbf{c}$, $\mathbf{f}$, and $\mathbf{i}$ have unique and independent streams of were still alive in the independent 
consciousness. lab. (However, the consciousnesses

crucial and significant (However, the crucial issue is what happened and significant issue is to the continuum what happened to the consciousness stream of continuum

$\mathbf{a}, \mathbf{b}, \mathbf{d}, \mathbf{e}, \mathbf{g}$, and $\mathbf{h}$, who consciousness streams of were in the lab until $\mathbf{T}_{\mathbf{1}}$ ?) $\mathbf{a}, \mathbf{b}, \mathbf{d}, \mathbf{e}, \mathbf{g}$, and $\mathbf{h}$, who were originally in the lab until $\left.\mathbf{T}_{1}\right)$ 


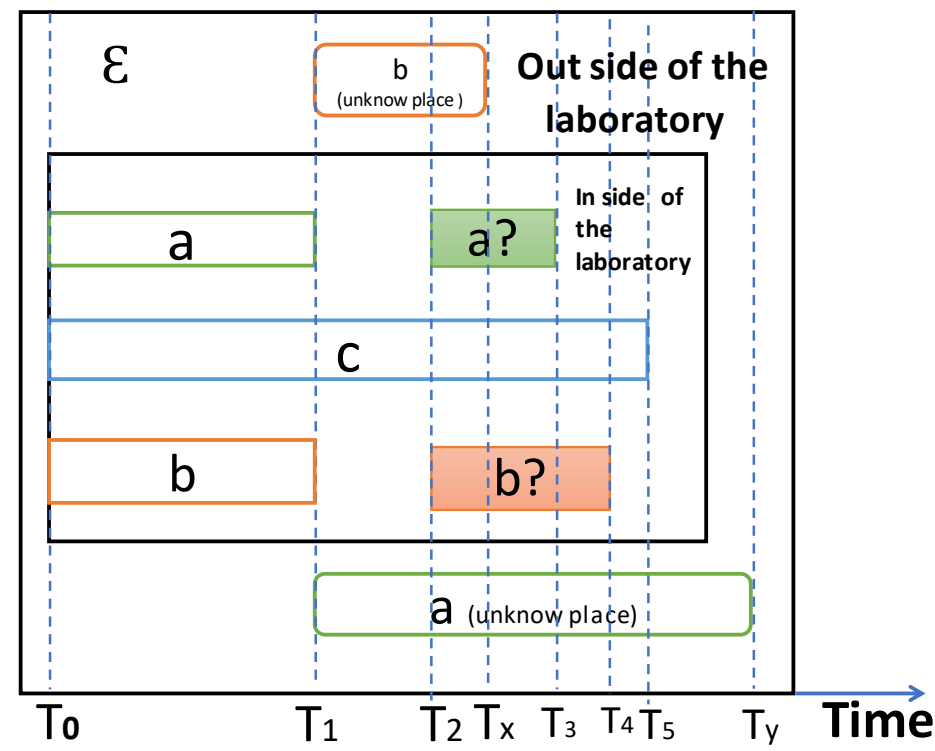

Venn diagram 1: The stream of distinctive continuum consciousness of $a, b$, and $c$ and their life span through time. Note: I demonstrate only one afterlife of $\mathbf{a}$ and $\mathbf{b}$ (Here, I only consider $\mathbf{a}, \mathbf{b}$, and $\mathbf{c}$ for easy reference out of nine original participants in the three experiments) of their continuum consciousness streams. All three streams of individual consciousness lived between $\mathbf{T}_{\mathbf{0}}$ and $\mathbf{T}_{\mathbf{1}}$ in the laboratory. Here, I suggest that after the death of ' $\mathbf{a}$ ' might be lived (afterlife, from $\mathbf{T}_{1}$ to $\mathbf{T}_{\mathbf{x}}$ ) and $\mathbf{b}$ lived from $\mathbf{T}_{\mathbf{1}}$ to $\mathbf{T}_{\mathbf{y}}$, outside (unknown places) of the lab that might be the only option to avoid logical contradictions. However, $\mathbf{c}$ might live $\mathbf{T}_{\mathbf{1}}$ to $\mathbf{T}_{\mathbf{5}}$ in the laboratory. Here, only demonstrated $\boldsymbol{a}$ ? and $\boldsymbol{b}$ ? (At $\mathbf{T}_{\mathbf{2}}$ ) who independently lived $\mathbf{T}_{\mathbf{1}}$ to $\mathbf{T}_{\mathbf{3}}$ and $\mathbf{T}_{\mathbf{1}}$ to $\mathbf{T}_{\mathbf{4}}$ in the lab were similarly nurtured. 


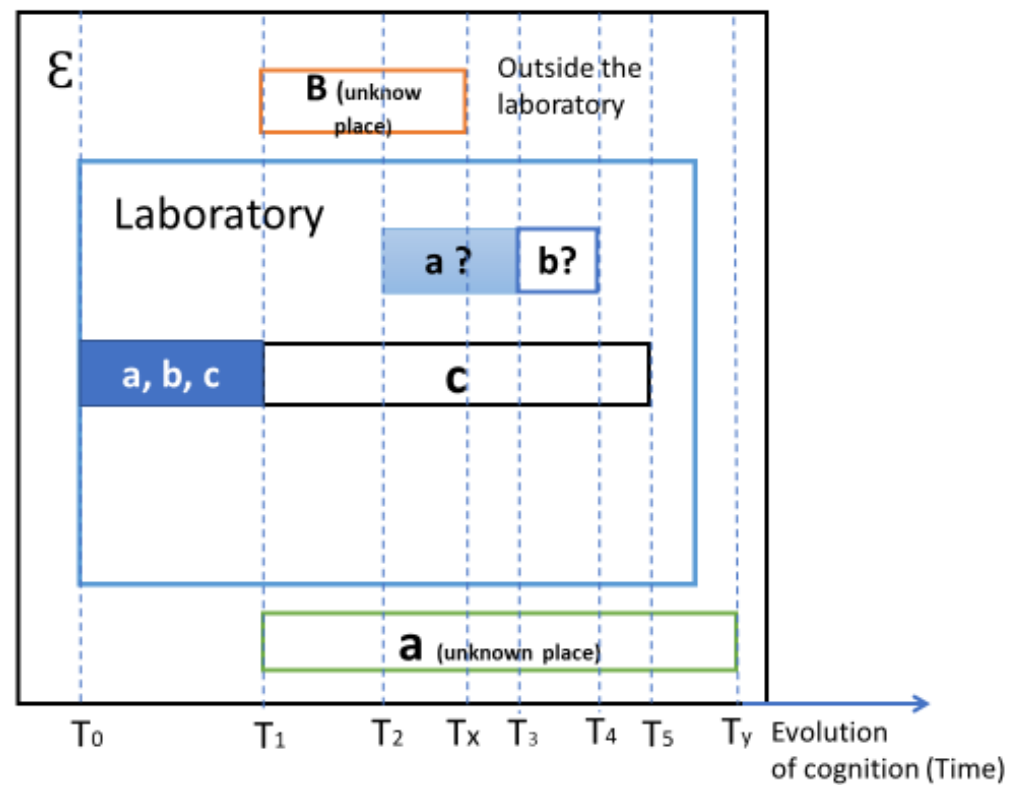

\section{Venn diagram 2:}

\section{The cognitive functions of $a, b$, and $c$ and their life span over time:}

Note: I demonstrate only one afterlife of $\mathbf{a}$ and $\mathbf{b}$ (out of nine participants in the three experiments) of their continuum consciousness streams. The laboratory's three streams of individual consciousness of a, b, and c lived between $\mathrm{T} 0$ and $\mathrm{T} 1$. Three of them had similar cognitive functions until $\mathbf{T}_{1}$. Here, I suggest that after the death of 'a' lived from $\mathbf{T}_{\mathbf{1}}$ to $\mathbf{T}_{\mathbf{x}}$ and $\mathbf{b}$ lived from $\mathbf{T}_{\mathbf{1}}$ to $\mathbf{T}_{\mathbf{y}}$, outside (unknown places) of the lab, that might avoid logical contradictions of results. However, $\mathbf{c}$ lived from $\mathbf{T}_{\mathbf{1}}$ to $\mathbf{T}_{\mathbf{5}}$ in the laboratory. The lives of frozen or artificially reconstructed brains of $\boldsymbol{a}$ and $\boldsymbol{b}$ are at $\mathbf{T}_{\mathbf{2}}$. The brain of 'a?' lived $\mathbf{T}_{\mathbf{1}}$ to $\mathbf{T}_{\mathbf{3}}$, and 'b?' lived $\mathbf{T}_{\mathbf{1}}$ to $\mathbf{T}_{\mathbf{4}}$ in the lab were 
similarly nurtured.

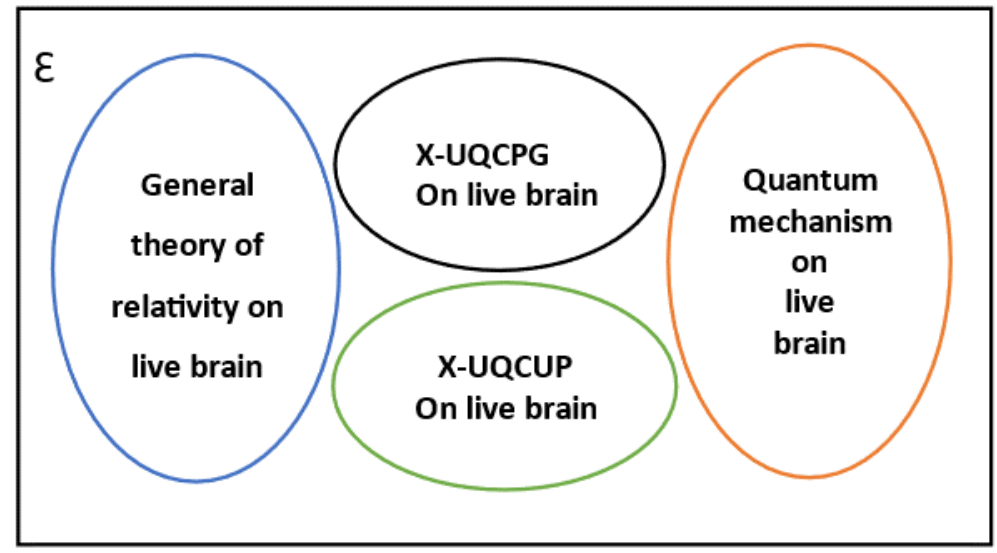

Venn diagram 3: This Venn diagram is a probable relationship between the consciousness of the human brain (or any other living being-life-), the theory of general relativity (GR), quantum mechanics, X-UQCGP, and X-UQCUP. Therefore, the union of four sets in the conscious live brain with symbols of the Venn diagram is as follows:

GR $\mathbf{U}$ X-UQCUP $\mathbf{U}$ X-UQCGP $\mathbf{U}$ Quantum mechanism = union of consciousness of a live brain. All four are disjoint sets:

GR $\cap$ X-UQCUP $\cap$ X-UQCGP $\cap$ Quantum mechanism $=\varnothing$ 


\section{General Discussion}

What happened to the consciousness of the brains of a, b, d, e, g, and h; from time $\mathrm{T}_{1}$ to $\mathrm{T}_{2}$ of the three experiments? How did brains gain 'new' consciousness at $\mathrm{T}_{2}$ ? Whom consciousness identities are now of new thirty-the brains? For example, how did the similar new eleven brains, (which) are identical to the brain 'a', start new consciousness simultaneously at $\mathrm{T}_{2}$ in the third experiment? It might be more convenient to understand the argument if any scientist or reader of this article could imagine that he and his identical two siblings of the triplets are participants in this research to analyze the results of the experiments. The third experiment is crucial to answering one of the research objectives. Someone can argue that the original a, b, d, e, g, and h among the thirty-three brains (or not) after $\mathrm{T}^{2}$ in the lab. For example, did the similar consciousness of 'a' exist among similar $a ? a^{1} a^{2}, a 3, b ? b^{1}, b^{2}, b^{3}, c, c^{1}, c^{1}$, and $c^{3}$ brains in the lab? If not, what happened to the consciousness of ' $\mathrm{a}$ ' in the lab before $\mathrm{T}_{1}$ ?

If the original person 'a' existed brain in the lab while all eleven brains were identical, how and why did the original 'a' select a particular brain out of eleven identical-similar brains? These are crucial and big questions that need to be solved here. Otherwise, 'a' should feel aware that 'a' 


\section{CONSCIOUSNESS AND AFTERLIFE}

simultaneously lived within two or more identical brains in the lab after $\mathrm{T}_{2}$.

Suppose Orch Or or any other materialistic theory might suggest that the original 'a' might be among those brains after $\mathrm{T}_{2}$. However, ' $\mathrm{a}$ ' has no life between $\mathrm{T}_{1}$ and $\mathrm{T}_{2}$. In addition, no stream of series of the afterlife might be their conclusion. However, they might not be smart enough to answer how or why 'a' (and 'b') were or were not among such perfectly identical eleven brains simultaneously made. Because the new life of twenty-seven and six brains (frozen) gains life at $\mathrm{T}_{2}$, it appears to emerge as in pig brains (Vrselja, Z. et al. 2019). Moreover, their current opinions of the afterlife make it challenging to identify who lives in each conscious brain. This article's argument might convince us that the new life in pigs' brains was probably not similar to "pigs" consciousness before specific brains death.

I propose that there are probably two or more or an infinite number of brains physically identical to any given brain simultaneously in the universe/s. Our introspections indicate that a person's consciousness has a unique continuum throughout life. Furthermore, we are generalizing our experience, and scientific findings suggest that the identity of consciousness would not exchange or move identical brains elsewhere or simultaneously. Therefore, there was no overlap or coincidence of similar feelings within two or more similar brains, which might create confusion.

One may propose that everyone has a universal, unique consciousness, a continuous stream of distinct consciousness, and no series of afterlife continuums. However, such a proposal would create contradictions again. 


\section{CONSCIOUSNESS AND AFTERLIFE}

If cognitive function applies to a Venn diagram one for experiment three, their cognition (above $\left.\mathrm{T}_{2}\right)$ will be:

\section{$a \cap b \cap c \cap a^{1} \cap a^{2} \cap a^{3} \cap b^{1} \cap b^{2} \cap b^{3} \cap c^{1} \cap c^{2} \cap c^{3}=X$ or cognitive}

functions of these twelve brains will be similar from time $\mathrm{T}_{2}$ and beyond in the laboratory.

According to these mathematical expressions, $\mathrm{X}$ depicts similarities in every aspect of identical brains' cognitive functions, except their unique-individual consciousness. The consciousness of 'a and 'b' (who were until $\mathrm{T}_{1}$ ) might not be similar persons of 'a'? and 'b'? after $\mathrm{T}_{2}$.

$\{a ? b ?\} \cap \mathrm{Lab}=\varnothing$

I did not arrange an additional experiment to find more precise facts on two microparticles to discuss the hypothesis in the results of this study. X-UQCGP (Dayathilake, 2017; Dayathilake, 2017; Dayathilake, 2017: Dayathilake, 2018) may carry the finally evolved (ultraquantum) 'key' genome when somebody or/an animal is dead, which may help bond and 'lock' with the new life. However, X-UQCGP (or X-UQCUP) might not be physically able to test in a laboratory unless the working hypothesis of theoretical and logical arguments along with scientific facts. However, thought experiments one, two, and three suggest that there may be naturally created two, three, more, or infinite physically identical brains in the universe/s and their similar 'keys' of X-UQCGP. Alternatively, if someone gets birth and their consciousness results from a coincidence, such coincidence might happen two or more or infinite times in the universe/s. Therefore, I suggest that to avoid similar multiple identical consciousnesses and universal confusion, X-UQCUP might 


\section{CONSCIOUSNESS AND AFTERLIFE}

naturally be created.

The materialistic aspect does not consider two kinds of such compounds of particles that emit and move to bond with a suitable zygote/primary nervous system/embryo at infinite velocity. Previously, physics discussed hypothetical particles tachyon (Feinberg, 1967) that possibly move faster than light. However, if such a mechanism does not exist, it will again contradict itself because there may be two, many, or an infinite number of identical consciousnesses. Materialists might find difficulties in explaining the results of the third experiment without the speculation of X-UQCUP and X-UQCGP. In other words, a (myself) and b (you) might be a continuum out of the lab after $\mathrm{T}_{1}$.

Both (X- UQCPG + X -UQCUP) particles may be bonded exceptionally and cannot break when justifying the hypothesis. However, I cannot precisely answer how those particles originate in the universe/s and why. Do they never destroy? Moreover, these two particles may not exist without live neurons over time. The combined two particles may not be discussed with either general relativity or quantum theory. Moreover, such particles may be emitted from a dead brain and simultaneously move at infinite speed to bond with another suitable prematurely vacant nervous system.

Furthermore, the observers or researchers in the lab might never find or face a significant challenge in identifying whether the similar stream of consciousness of 'a' and 'b' continues in new brains after $\mathrm{T}_{2}$, out of eleven identical brains. Scientists need to apply the results of three experiments 


\section{CONSCIOUSNESS AND AFTERLIFE}

logically. Otherwise, the confusion will continue.

Nevertheless, any person's consciousness continues in the live brain until death; in other words, the living brain is not a zombie like a computer. To Hawking, the live human brain is similar to a zombie (unconscious) computer. He might assume that consciousness has no such unknown (such as X- UQCUP) particle, which quantum theory might not explain. Moreover, it may be a momentby-moment manifestation of the mind, which is said to happen in every person all the time. (Karunamuni, 2015). Moreover, human consciousness flows like a stream governed by five characteristics (James, 1890).

In other words, materialists may say that participants' lives were a continuum from $\mathrm{T}_{0}$ to $\mathrm{T}_{1 \text {, which }}$ is an empirical fact. Nevertheless, there was no afterlife from $\mathrm{T}_{1}$ to $\mathrm{T}_{2}$, and the similar original consciousness of the six regained similar consciousness and cognitions at $\mathrm{T}_{2}$ in the lab. However, they will be unanswerable to the results of the third experiment; if someone asks them to show the brain of 'a' out of eleven identical brains, they will be in trouble. Furthermore, if they say 'a' was neither in nor out of the lab, they cannot answer why. Nevertheless, the only option is that 'a' might live from $\mathrm{T}_{1}$, elsewhere outside the lab.

We may assume that the reference to present life uniqueness of self-awareness might be a continuum from childhood (probably from an early embryo) until death. In other words, in the development of a given person's brain in size and its neural organization, new matter replaces inside or outer neurons of the brain (such as new proteins, evolving DNA, neuroplasticity, and neurogenesis) or shrinks in age, still specific - unique consciousness continuum with time. 


\section{CONSCIOUSNESS AND AFTERLIFE}

Therefore, if the six brains did not die but minimized or neutralized (a reference to experiment one) their consciousness at $\mathrm{T}_{1}$, they would continue their unique psychological awareness from $\mathrm{T}_{2}$ and beyond. Nevertheless, if these six participants indeed die, researchers face a significant challenge to find the original consciousness of a, b, d, e, g, or ' $h$ ' consequently; however, a problematic issue seems essential to find what might happen to our continuum consciousness after death at $\mathrm{T}_{1}$. If materialism is acceptable, no new physics and no afterlife are involved. However, the issue is why six previous persons were not born at $\mathrm{T}_{2}$ among the thirty-three brains. Suppose one can argue that there is a possibility to be born again among thirty-three while keeping a time interval of $T_{1}$ to $T_{2}$. If those six were born again among thirty-three, one could question materialists in which specific brains previous life of six were born and why. Moreover, one can ask materialists (when they say similar consciousness will arise in a similar brain) how specific consciousness, before death, will select six specific-distinct brains among the several identical brains.

If scientists assumed that pig brains (Vrselja et al., 2019) regained similar 'unique' consciousness in (their empirical experiment), similar brains before death after being frozen might be their fault judgment. Analyzing the results of the third study creates contradictions with a particular conclusion. Furthermore, even identical brains are structural, biological, clinical, neurological, cognitive, psychological, and physically similar; however, consciousness is unique in a specific person. Therefore, lab researchers face trouble finding answers, such as where I am -' $\mathrm{a}^{\prime}$ - indeed lived after death or whether in a similar eleven brain of $\mathrm{a}^{1}, \mathrm{a}^{2}, \mathrm{a}^{3},{ }^{1}, \mathrm{~b}^{2}, \mathrm{~b}^{3}, \mathrm{c}^{1}, \mathrm{c}^{2}, \mathrm{c}^{3}$, along with frozen dead brain of 'a' and 'b.' Furthermore, did 'a's consciousness live somewhere in the universe/s 


\section{CONSCIOUSNESS AND AFTERLIFE}

or not?

Therefore, materialism or quantum mechanics alone might not answer the above issues. Alternatively, in other words, unknown matter (X-UQCGP) may be involved here. Here, I cannot precisely discuss the X-UQ particles and evidence of present knowledge of biophysics or other physics theories. However, the such unidentified matter might closely function with a quantum particle in brain neurons, and the functions might depend on the Orch Or theory.

Quantum mechanics might not adequately discuss the such tiny matter in size, mass, speed, velocity, or time. If such particles exist, it is not always necessary for them to behave according to quantum mechanics. From a mathematical aspect, although one is a natural number, it does not present an absolute number (quantity). Nevertheless, it indicates relative measurement (e.g., one light-year, kilo, or nanometer). Regardless, in any natural number, $a$ between zero and 1 (one) has a decimal representation of relative quantities with an infinite decimal.

Moreover, it is unclear whether such absurdly tiny scales have any physical meaning (Roger, 1989). Therefore, asking for the smallest or least in mass particle or/and most minor time fracture seems meaningless. Here, I argue that if there are countless smaller particles in size and different new physical qualities, they might not behave according to the laws in the present knowledge of physics. Those might be beyond direct empirical research, such as any elementary - subatomic particles. I use this mathematical application to assume the probability of the existence of particles smaller than empirical elements already found by physicists. Here, I use these mathematical 


\section{CONSCIOUSNESS AND AFTERLIFE}

thoughts to suggest the two tiny particles I have already mentioned. Otherwise, when it travels through massive bodies such as black holes or colossal stars, it would also be destroyed, deviated, or attached to them by gravity. (Dayathilake, 2018). Since electromagnetic waves and quantum particles have space-time curvature, such particles cannot pass through these massive bodies in the universe/s and have an absolute speed of $3 \times 10^{8} \mathrm{~ms}^{-1}$. Nevertheless, ultraquantum particles (theory) assume that those particles have infinite speed and are massless, so space-time has no curvature. However, without (firm) evidence, I suggest that those particles simultaneously have a multi(or infinite) dimensional movement within the live brain at infinite speed.

Consequently, the life of the nervous system might be formed by union with two unidentified microparticles and travel in infinite velocity from one dead brain to a new vacant primary nerve system. Data show that subatomic particles break light speed (Eugenie, 2011) and quantum entanglement (Schrodinger, 1935), encouraging my idea of infinite velocity. I call it an (unknownX) ultraquantum consciousness unique particle (X-UQCUP), which would be universally unique to any given person or/and animal. According to this hypothesis, there are no two or more XUQCUPs in living beings elsewhere in the universe/s; therefore, there are no similar consciousness identities.

Neurobiological changes may impact quantum mechanics and be minimal, inactive, neutral, or less conscious. For example, if there is a lack of oxygen, glucose, and general anesthesia, such fluctuations of consciousness might occur. Here, I explain how consciousness might exist in the brain with the direct results of three experiments. I propose that infinite movement of (X- 


\section{CONSCIOUSNESS AND AFTERLIFE}

$\mathrm{UQCUP}+\mathrm{X}-\mathrm{UQCGP}$ ) in a specific brain's active areas of a person may result in present-moment awareness of consciousness. The evolution of X-UQCGP may depend on the physical brain function of a particular active area/s. X-UQCGP might exist in the whole live brain simultaneously. Therefore, the speed of thoughts might depend on the neuronal network's operating speed, although (X-UQCUP + X-UQCGP) may have infinite speed outside (multi or infinite) dimensional (simultaneous) vibration and exist as a 'cloud' in the entire live brain. Therefore, the 'cloud' size may be expanded while developing the brain. Here, I would emphasize that bonded particles do not represent the notion of a spiritual soul that has been told particular and ever-suffering or happy birth after death and independent of brain functions, which has no scientific rationale.

The third theoretical experiment attempts to make exact brains develop in completely similar nurtures. (1) a physical foundation of the brain is a scientific fact, (2) we, billions of healthy humans on earth, the experience that our consciousness continues from past to present, and it is unique to each of their life awareness-consciousness-existence, (3) cloning identical animals or human is a fact-possible in present science and technology (4) already there may be numerous physically identical brains may exist in the universe/s, such as to similar cloning humans and animals. (Because astronomers suppose there are nearly 100 to $200 \times 10^{21}$ - approximately 200 billion trillion stars- in 'our' universe. I suggest that more or infinite numbers of universes might exist in infinite space (Dayathilake, 2017; Dayathilake, 2017; Dayathilake, 2017: Dayathilake, 2018). Scientists claim that billions of stars might already have possible planets where life exists 


\section{CONSCIOUSNESS AND AFTERLIFE}

in our universe. (5) Quantum and GR theories do not give a rational answer with materialistic aspects. Simultaneously, reductionists did not find unique empirical-physical matter in each brain to justify consciousness.

I analyzed the results in the first table, and Venn diagrams one and two for an acceptable answer, especially in experiment three.

(6) The latest research on consciousness, such as Orch Or theory (Hameroff \& Penrose, 2014), or any other, might not be able to challenge the argument here of three experiments.

Because their hypotheses may not be strong enough to discuss what happened to 'I-me-myself' (a) $\operatorname{you}^{1}(\mathrm{~b})$ or $\operatorname{you}^{2}(\mathrm{c})$ individual continuum consciousness in the lab. In other words, what happened to three of their consciousness ('I-me-myself' (a), you ${ }^{1}(\mathrm{~b})$, and $\mathrm{you}^{2}$ (c)? (because no one existed between $\mathrm{T}_{1}$ and $\mathrm{T}_{2}$ ). Therefore, whom consciouses existed in the lab after $\mathrm{T} 2$ (within eleven similar identical brains)? Who were actually in the new eleven identical brains in the lab? According to my suggestion, it might be clear that myself-I' (a), you ${ }^{1}(\mathrm{~b})$, and you ${ }^{2}(\mathrm{c})$ might not exist in the brains of those eleven identical brains ( $a$ ? $b$ ? and $a^{1}$ to $c^{3}$ ) simultaneously. Otherwise, (for example), myself(a), and you(b) would have been in all eleven (similar) brains simultaneously. However, it might not happen, and contradiction. Furthermore, who was in the new eleven brains after $T_{2}$ in the lab? These questions might not explain other than my points of one to six above. (7) As I previously said, a universally X-UQCUP continuum is a stream from birth to death and the afterlife. Moreover, no healthy person is simultaneously confused with 2, 3, or more similar lives 


\section{CONSCIOUSNESS AND AFTERLIFE}

and multi-awareness (multiconsciousness). Therefore, a person's consciousness contradicts unless we do not apply the X-UQCUP of this theory.

(8) Nevertheless, if the consciousness of life emerges just as a rare accident without continuum afterlives and with a purely physical effect. Similar accidents might also occur in the past and future between present life. However, no researcher might accept that such a coincidence occurs every time with a time gap between the past, present, and future life's existence. If a similar person's life gains two or more places simultaneously due to (just) coincidence, the materialistic argument fails again with multiple identical consciousnesses. Therefore, you, me, or any other might confuse about multiple existences simultaneously in many places in the universe if life is just a result of a coincidence (9). Therefore, if life is just the result of a coincidence of only known and empirical physical matter, it cannot solve the problem. (10). Nevertheless, point nine will be a contradiction; if such two, more, or infinite similar coincidences might happen simultaneously, similar individuals may be born with identical consciousness (but not unique); in other words, we should feel that we are concurrently in two or more or infinite places simultaneously. (11) Most importantly, I assume I naturally attempted to avoid such universal self-confusion. However, the nature of matter might naturally originate carrier particles of individual consciousness (unknown -X unique particle) and continuum stream of consciousness in the afterlife (might be with natural responsibility). However, it is too early to suggest whether this purpose of unique consciousness has any relationship with life in the universe/s. To avoid those contractions and three experiment results, I suppose there is no time gap to travel to X-two combined microparticles (X-UQCGP and 


\section{CONSCIOUSNESS AND AFTERLIFE}

X-UQCUP) between the dead brain and new life in a primary nervous system. Therefore, there might be no issue with distance travel between those two environments of the dead brain to the vacant nerve system. (13) I emphasize that one, two, or more (X-UQCGP) with a similar 'key' may emit at any time. (14) Nevertheless, there may be many more vacant similar nervous systems than the number emitting any X-UQCGP at any given time. In other words, there may be more or infinite vacant and matching nervous systems in the universe/s than any given number of similar 'keys' of X-UQCGP(+ X-UQCUP)s that might emit at any given time. However, here I should emphasize that if there may be two or more beings having a similar' key.' However, I may not suggest that there are two or more beings with similar X-UQCGP, except for the 'key - ultra quantum gene' of X-UQCGP.

Therefore, the evolution of life in the universe/s and consciousness might not be merely a result of known physical matters of the brain and a just outcome of coincidence, as materialism explains. However, it might result from phenomena only discussed with new physics and probably beyond empirical studies. Otherwise, the principle of individual-unique consciousness of life theory cannot apply. In other words,' me/I,' you ${ }^{1}$, you ${ }^{2}$ might experience two or more identical brains simultaneously at any given moment (in diverse areas of the universe/s), as I have demonstrated in research observations after $\mathrm{T}_{2}$.

Here, the X-UQCGP might be changed by the brain's quantum particles. Both combined microparticles may not move to any other brain or beyond the specific brain until death. In other words, when a person's brain has a velocity relative to any external matter, the 'cloud' of two 


\section{CONSCIOUSNESS AND AFTERLIFE}

ultraquantum particles might move simultaneously with the brain. In other words, the two particle sizes may be similar to the live brain area at any given moment because the two particles move simultaneously at an infinite velocity in the regions of the entire live brain. X-UQCGP may not affect changes that evolve (positively or negatively) in the physical brain. In other words, the evolution of X-UQCGP in the brain depends on nature, nurture, biology, biophysics, and related behavior. Therefore, the total evolution of these factors may impact the positive or negative effects of X-UQCGP. One may suggest that those particles act as an independent soul.' However, if there is an independent soul, such as a 'constant matter' in identical twins or triplets (nurtured similarly), it should have a variation of I.Q. and behaviors. X-UQCUP might not deviate from X-UQCGP or any person's materialistic brain, which continuously makes its stream of a unique individual consciousness. Therefore, X-UQCUP might never change over time in a particular life and might continue a unique consciousness even after death. However, the ever-evolving X-UQCGP in a specific brain and the characteristic final 'key gene/s' of evolution may be crucial to selecting and bonding the next life.

I suggest additional theoretical evidence of a single unique 'cloud of the two microparticles' of any living brain(areas) in humans or animals. For example, billions of neurons in a human brain are not linked as a single network; there are always gaps- space between each other by synapse of every neuron and no unbroken microtubule links (a single network) within the entire brain. Therefore, it is difficult to make a possible argument for a single individual identity in one brain without the theory mentioned here. If we do not consider this hypothesis, one can argue that there 


\section{CONSCIOUSNESS AND AFTERLIFE}

might be billions of individuals - independent materialist persons - (therefore billions of separate consciousnesses) in a single brain, and why not so.

I use split-brain research findings to strengthen my idea of the new physics 'matter' of two combined microparticle hypotheses. Suppose researchers on split brains suggest multiple modules. In that case, the brain is composed of hundreds of independent centers of thought called "modules" (Blakeslee, 1996), two minds in one person (Schiffer, 2021), leading to the conclusion that simple dual consciousness (i.e., right-brain/left-brain model of the mind) is a gross oversimplification and that the brain is organized into hundreds or perhaps even thousands of modular-processing systems. (Gazzaniga, M., LeDoux, J., 1978; Gazzaniga, M., 1985). However, they are not yet able to make a unified theory to suggest how the material brain is responsible for origin and continuum (at least in the present life span) as a universally unique you (or me) within two, more, or infinite identical brains, if in the universes in diverse nurture, without my theory of two microparticles. They do not yet suggest how universally unique individual self-consciousness-awareness-feeling is, with merely materialistic brain function. My thought experiment point is just mentalistic, which can explain relativity theory and the quantum mechanism of brain matter to solve how consciousness might exist in the brain without assuming my view. Second, there are not just two major apart hemispheres in different which have distinctive functions, but billions of apart neurons with micro-specific functions unite as we experience in a single self-person-you or me in a single brain among two or many possible apart brains in the universe/s. My alternative principle suggests that two hemispheres and billions of neurons unite for a unique individual, as already explained. 


\section{CONSCIOUSNESS AND AFTERLIFE}

Third, split-brain research convinces us that (if) microparticles contribute a significant role in unique consciousness. This combined microparticle does not impact (in this point, microparticle function neutrally on brain biology) physical matter (for example, they communicate in coordination with each other neuron to the brain), such as the materialistic corpus callosum and the physical matter of the brain. Which impact microparticles make (for example) different feelingawareness as we (might) evolve (+/-), or change the nature or nurture of brain and behavior as some reincarnation work suppose remind there past life. However, I cannot strongly oppose their argument because if reincarnation results are scientific facts, microparticle genomes rarely might deviate and impact the brain, recalling memories.

Accordingly, no alternative theory has yet been seen that may challenge this argument about the afterlife. Therefore, as Hawking has discussed, we cannot compare a significant afterlife question with broken computers because computers do not have life and continuum consciousness but are just materialistic machines. Moreover, reincarnation can save Schrodinger's cat (Merali, 2008), which may strengthen this theory.

The phenomena of X-UQCGP could naturally evolve positively (+) or negatively (-), impacting the nature and nurture of the person's brain (Dayathilake, 2017; Dayathilake, 2017; Dayathilake, 2017: Dayathilake, 2018). Moreover, the notion of a specific and eternal soul independent of brain functions contradicts while observing behaviors and thoughts of persons with Alzheimer's disease, mental disorders, aging (Dayathilake, 2017), and behaviors. If humans have such an independent soul, patients' behaviors or other cognitive functions do not deviate from whatever brain matter 


\section{CONSCIOUSNESS AND AFTERLIFE}

makes them vary. In other words, if there is such a permanent and independent soul, neurological or psychiatric patients may not suffer from disorders of their physical brain. Therefore, I suppose there is also no free will (Dayathilake, 2017; Dayathilake, 2017). MV scanning (meditation) by healthy mind viruses might influence the evolution of their intelligence. If a person can scan mind viruses successfully, his brain-mind evolves (+) positively, or if scanning is not robust enough, it will be negatively(-) as depicted in a 3D graph (Dayathilake, 2017; Dayathilake, 2017; Dayathilake, 2017: Dayathilake, 2018) evolving, along with nature, nurture, and time. Therefore, such MV scanning may impact $( \pm)$ X-UQCP natural evolution. I found more than 27,638 peer review studies for keyword searches on meditation in PubMed Central on diverse research titles. Moreover, a study found that loving-kindness meditation may help to improve subjective wellbeing (Chao, 2020), and 1917 research articles discussed loving-kindness meditation.

When a successful MV scan evolves the intelligence of a given person's intelligent decisions, when scanning, MV might naturally reward psychological well-being. If decisions are harmful (inter- or intrapersonal), such decisions might increase the risk of psychological suffering (Dayathilake, 2018). A study showed that once a nerve becomes electrically active, it can influence the genes, influencing how the nerve develops (Gazzaniga, 1994). Therefore, consciousness and the brain have a close relationship. Although nature and nurture influence the I.Q. of adults (Campbell, 1994). Consequently, I assume that HMV — highly activated persons with relatively few and weaker MV intelligence decline with age and might be deficient (Dayathilake, 2017; Dayathilake, 2017) — and research has indicated that clever brains age more slowly (Rabbitt et al. 2003) 


\section{CONSCIOUSNESS AND AFTERLIFE}

These hypotheses might not ultimately discuss the theories. However, any given person or animal has a unique consciousness, which is a primary principle of the universe and might be a continuum after death. The brain might strongly bond with these two unknown ultraquantum particles, regardless of whether the brain develops in size, damages, splits, shrinks, ages, and their unique consciousness continuum until death. Moreover, those X-two microparticles might not impact psychological qualities in the physical brain. Moreover, other physical-material, neurological, and psychological chemicals, nutrition, anesthetics, drugs, and characteristics of the remaining XUQCGP might impact the quality and quantity of emotions and level of consciousness awareness.

Nevertheless, this may begin a different methodological approach for consciousness and afterlife studies. If we can find more empirical facts strengthening the theory further, it might help evolve our global unity, peace, health, happiness, and many other facts toward making a better world. These findings may naturally emphasize to humankind how risky the journey of the universe/s we are in (Dayathilake, 1991), why we need to learn and practice from real intellectuals, and how to scan our MV by HMV (Dayathilake, 2017; Dayathilake, 2017). Such intellectuals and scientists may encourage or properly program people's minds and behaviors (Dayathilake, 2017; Dayathilake, 2017), supporting these research findings. Here, I have shown a few inter- and intrapersonal biological networks that impact the evolution of personnel and global intelligence and psychological well-being. However, I need to minimize exaggeration; the consciousness continuum of the afterlife and intelligence evolution may affect personal, global, and universal goals for the survival of psychological well-being beings. Strong determinism (Penrose, 1989) and 
the afterlife hypothesis also do not seem contradictory. However, it is not easy to precisely find the natural purpose of the unique consciousness continuum in the evolution of intelligence via the universe/s. Alternatively, I suppose we might find facts in the future on more robust hypotheses to strengthen my study. In that case, humankind may naturally attempt to find better methods to evolve their X-UQCGP for a happier life on earth positively and be born in more comfortable places after their death in the universe/s by evolving their intelligence positively over time. 
Acknowledgments:

My sincere thanks for the tremendous respect for my dearest father, Mr. K.L. Senarath Premathilake (late), and mother, Mrs. K.L. Indra Kurulugama (late), who has given not only nature but also nurture of their best, and my immense gratitude to K.L. Martin (Late grandfather), Mrs. Punchimanike Dalukdeniya (late grandmother), Mr. K.L. Bandula (late grandfather) K.L. Podimanike (late grandmother) nephews- Sanidu Upamal Karunaratne, Nishal Paranagama, Sandaru Shamaindra Senarath, K.L.S. Kawya Kaushalya Kurulugama, and nieces - Ushara Dulakshi Karunaratne, Nishali Paranagama, Mr. and Mrs. Ekanayake and family; option Professor H.M. Petry, committee members, and all the staff, Prof. John Nicholls, Prof. Kenneth Muller, H. E. Mahinda Rajapaksa, H. E. Gotabaya Rajapaksa, Prof. Carlo Fonseka (late), Dr. N Fernando, Sir Arthur C. Clarke (late), Prof. Y. Karunadasa, Mr. H.B. Jayewardene (late), Mr. W. G. Rodrigo (late), Mr. Douglas Perera 
(late), Dr. Hashitha Mahen Dombagahawatta, Mr. Sanjeewa Wickramanayake, Mrs. Probodhinee Marasingha, and others who encourage and assist.

\section{Reference}

Alexander, E., (2012). Proof of Heaven. (Simon \& Schuster, New York).

Berkeley, G.,(1975). Philosophical works (Ayers, M., editor).London: Dennett 


\section{CONSCIOUSNESS AND AFTERLIFE}

Blakeslee, T., (1996). Beyond the Conscious Mind. Unlocking the Secrets of the Self. pp. 6-7. ISBN 9780306452628.

Campbell, P., (1994). What if intelligence is inheritable? Nature 371,637.

Chalmers, D.J.,(2012). Constructing the world. New York (N.Y.): Oxford University Press.

Chao Liu, Hao Chen, Chia-Yi Liu, Rung-Tai Lin, and Wen-Ko Chiou, (June 2020) The Effect of Loving-Kindness Meditation on Flight Attendants' Spirituality, Mindfulness, and Subjective WellBeing Healthcare (Basel).; 8(2): 174. Published online. 16. DOI: 10.3390/healthcare8020174

Chopra, D., (2001)How to know God: the soul's journey into them the mystery of mysteries. New York: Running Press Book Publishers

Chopra D., (2006). Life after Death: The Burden of Proof (Harmony, New York).

Dayathilake, K.L.S., (2017). Nature of Human Intelligence. Available at SSRN: https://ssrn.com/abstract=3848408 or http://dx.doi.org/10.2139/ssrn.3848408

Dayathilake, K.L.S., (June 16, 2017) A Multidisciplinary Hypothesis to Reincarnation: Does It a Universal Survival of the Genomic Particle of the Consciousness When a Brain Death? https://papers.ssrn.com/sol3/papers.cfm?abstract_id=2986253

Dayathilake, K.L.S., (July 12, 2018). Life after death in the evolution of intelligence. https://psyarxiv.com/2hkw4/Preprint DOI 10.31234/osf.io/2hkw4 


\section{CONSCIOUSNESS AND AFTERLIFE}

Dayathilake, K.L.S., (2017). Nature of human intelligence evolution on psychological well-being. doi:10.17605/OSF.IO/T8CQN,//psyarxiv.com/t8cqn/

Dayathilake, K.L.S.,(Feb.15, 1991). 'Scientific foundation for optimum happiness of invaluable and extraordinary humanity.' National Archives collection, Sri Lanka.

Dennett, D.C.,(1991). Consciousness Explained. Boston (M.A.): Little Brown.

Dennett, D.C.,(1995). Darwin's dangerous idea: evolution and the meanings of life. New

York (N.Y.): Simon and Schuster.

Dennett, D.C.,\& Kinsbourne M., (1991).Time and the observer: the where and when of consciousness. Behave Brain Sci; 15:183-247.

Eugenie, R. S., (2011). Neutrino-experiment-replicates-faster-than-light-finding-1.9393\#auth-1 Nature.452.

Facco, E. \& Agrillo, C., (2012) Near-death experiences between science and prejudice. Front Hum Neurosci, 10.3389/fnhum.2012.00209.

Feinberg, G. (1967). "Possibility of faster-than-light particles." Physical Review. 159 (5): 1089_ 1105. Bibcode:1967PhRv..159.1089F. doi:10.1103/.

Gazzaniga, M.S., (1994). Nature's Mind (Happer Colling Publishers, inc.).

Gazzaniga, M., LeDoux, J.,(1978). The Integrated Mind. Springer. pp. 132-161. ISBN 9781489922069. 
Gazzaniga, M., (1985). The Social Brain. Discovering the Networks of the Mind. pp. 77-79. ISBN 9780465078509.

Gombrich, R., (December 31, 2009). What the Buddha thought. Oxford Centre of Buddhist Studies Equinox Publishing Limited.

Hameroff, S.R. \& Penrose, R., (1996). Conscious events as orchestrated space-time selections. J Conscious Stud; 3(1):36-53.

Hameroff, S., (1998). Quantum computation in brain microtubules? The Penrose-Hameroff "Orch OR" model of consciousness. Philos Trans R Soc Lond Ser A, Math PhysSci;356:1869-96.

Hameroff, S.,(2007). The brain is a neuro-computer. Cogn Sci; 31:1035-45.

Hameroff, S., \& Penrose R., (2014). Consciousness in the universe A review of the 'Orch OR' theory Physics of Life Reviews 11 39-78(2014).

Hameroff, S.R. \& Penrose, R., (1996). Orchestrated reduction of quantum coherence in brain microtubules: a model for consciousness. In: Hameroff SR, Kaszniak AW, Scott AC, editors. Toward a science of consciousness; the first Tucson on discussions and debates. Cambridge (M.A.): MIT Press;.p.507-40. Additionally, published in Math Computer Simul 1996; 40:453-80.

Hameroff, S., (1998). Fundamentality: is the conscious mind subtly linked to a basic level of the universe? Trends Cogn Sci; 2:119-27. 


\section{CONSCIOUSNESS AND AFTERLIFE}

Hameroff, S. \& Penrose, R., (2014). "Consciousness in the universe." Physics of Life Reviews. 11 (1): 39-78. Bibcode: 2014Ph L Rv.11...39H. doi:10.1016/j.plrev.2013.08.002. PMID 24070914.

Hawking, S.W.,(2006). The Theory of Everything, Phoenix Books, ISBN 81-7992-591-9.

Hawking, S.W., (May 15, 2011). The Guardian.

James, W., (1890). The Principles of Psychology, Volume 1. H. Holt. p. 225.

Kant, I.,(1998). Critique of pure reason (Guyer P, Wood A.W., Trans.) Cambridge University Press.

Karunadasa, Y., (2010). The Theravada Abhidhamma. Its inquiry into the Nature of Conditioned Reality, p. 262. Centre of Buddhist Studies, The University of Hong Kong. ISBN 978-988-992966-4.

Karunamuni, N.D., (May 2015). "The Five-Aggregate Model of the Mind.” Sage Open. 5 (2): 215824401558386. doi:10.1177/2158244015583860.

Long, J., \& Perry, P., (2010). Evidence of the Afterlife: The Science of Near-Death Experiences 29 (Harper One, New York).

Machine, W., (2013). on Anatta, Encyclopedia Britannica.

Merali, Z., (2008). Reincarnation can save Schrödinger's cat. Nature 454, 8-9. 


\section{CONSCIOUSNESS AND AFTERLIFE}

Mobbs, D., \& Watt, C., (2011) There is nothing paranormal about near-death experiences: How neuroscience can explain seeing bright lights, meeting the dead, or being convinced you are one of them. Trends Cogn Sci 15 (10):447-449. CrossRef PubMed Google Scholar.

Parnia, S. et al., (January 16, 2019). AWARE—AWAreness during REsuscitation—A prospective study" Journal of European Resuscitation (DOI:http://dx.doi.org/10.1016/j.resuscitation.2014.09.004).

Penrose, R., (1989). The New Emperor's Mind Oxford University Press. ISBN 0099771705.

Penrose, R. \& Hameroff, S.R., (1995). What gaps? Reply to Grush and Churchland. J Conscious Stud; 2:98-112.

Penrose, R. \& Hameroff, S.,(2011) Consciousness in the universe: neuroscience, quantum spacetime geometry, and Orch OR theory. J Cosmol. 14.http://journalofcosmology.com/Consciousness160.html. 28.

Rabbitt, P., Chetwynd, A., McInnes, L.D.O., (Feb. 2003). clever brains age more slowly? Further exploration of nun results. Brit. Jour. Psychol.

Schiffer, F., (2021). Of Two Minds: The Revolutionary Science of Dual-Brain Psychology, 2nd, revised edition: 978-0684854243

Schrodinger., E., (2015). Oxford dictionary of physics. Seventh edition. 461p. 


\section{CONSCIOUSNESS AND AFTERLIFE}

Sutherland, S., (1989). "Consciousness." Macmillan Dictionary of Psychology. Macmillan. ISBN 978-0-333-38829-7

Thonnard, M., et al. (2013) Characteristics of near-death experiences memories as compared to real and imagined events memories. PLOS ONE 8(3):e57620. CrossRef PubMed Google Scholar.

Van Lommel, P., (2011) Near-death experiences: The experience of the self as real and not an illusion. Ann N Y Acad Sci 1234 (1):19-28. CrossRef PubMed Google Scholar.

Vrselja, Z.,et al. (2019). Nature 568, Restoration of brain circulation and cellular functions hours postmortem. Nature 568, 336-343.

Wegner, D.M.,(2002). The illusion of conscious will. Cambridge (M.A. ): MIT Press.

Whitehead, A.N., (1929). Process and reality. New York (N.Y. ): MacMillan; 27

Whitehead, A.N.,(1933). Adventure of ideas. London: MacMillan;

Zeman, A.,(2008). A Portrait of the Brain, Yale University Press Publication, ISBN 978-0-30011416-4. 